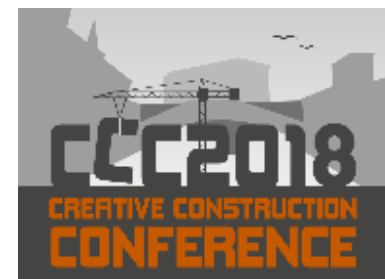

Proceedings of the Creative Construction Conference (2018)

Edited by: Miroslaw J. Skibniewski \& Miklos Hajdu

DOI 10.3311/CCC2018-099

Creative Construction Conference 2018, CCC 2018, 30 June - 3 July 2018, Ljubljana, Slovenia

\title{
Employing Critical Chain and Lean Concepts to Develop the Planning and Control Framework for Linear Construction Projects
}

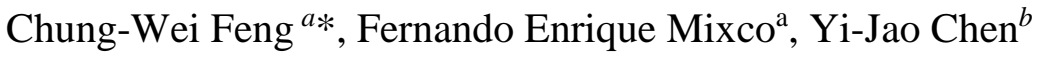 \\ ${ }^{a}$ National Cheng Kung University, \#1 Universioty Road, Tainan, Taiwan \\ ${ }^{b}$ National University of Kaohsiung, \#700 University Road, Kaohsiung, Taiwan
}

\begin{abstract}
A framework to plan and control construction projects by incorporating the concepts of critical chain, linear schedule, and lean construction is presented. Construction project managers need to seek the effective interaction between the variables of time and money to ensure the development and compliance of the project within the planned schedule and the budget limit. Such an interaction could further be complicated by the development of project activities involving multiple stakeholders such as specialized subcontractors in different areas of construction. Consequently, this multi-participation consists in determining the start and end of all activities carried out by different parties, as well as variations in productivity each subcontractor possesses, which greatly increases the risk of project delay and over budget. Although various methodologies have been employed to prevent such problems, lacking of control mechanism and risk management lead to ineffective practice in the real project.

This framework initially integrates CCPM (Critical Chain Project Management) and LSM (Linear Scheduling Method) to create a project buffer able to control the project in terms of time. The project buffer is obtained through the reduction of the duration of some activities belonging to the critical chain through the bonus-penalty system. In Addition, a cost buffer is included within the framework to encourage subcontractors to improve their productivity and to ensure continuity of the work, respectively. Furthermore, this study incorporates concepts from Lean Construction and EVM (Earned Value Method) for the purposes of (1) managing construction projects with a higher planning reliability and (2) monitoring and controlling the consumption of project buffer and cost buffer according to established consumption criteria.
\end{abstract}

Keywords: ;Critical Chain ; Lean Construction ;Linear Scheduling

\section{Introduction}

Construction project managers need to seek the effective interaction between the variables of time and money to ensure the development and compliance of the project within the planned schedule and the budget limit. Such an interaction could further be complicated by the development of project activities involving multiple stakeholders such as specialized subcontractors in different areas of construction. Consequently, this multi-participation consists in determining the start and end of all activities carried out by different parties, as well as variations in productivity each subcontractor possesses, which greatly increases the risk of project delay and over budget.

Different methodologies have emerged for effectively planning and controlling a project. The Critical Chain Project Management (CCPM) [1] focuses on eliminating the floats from activities and reallocating them into strategic positions on the critical chain. These time positions are known as "buffers". Figure 1 shows a comparison between a CPM (Critical Path Method) schedule and a CCPM schedule, where along with a reduction of the project duration includes a project buffer at the end of the project that extends to finish at the same time as the original schedule. Then, a buffer 
management process is implemented to control the buffer consumption throughout the life cycle of the project. This project buffer has a primary objective to serve as a control mechanism of the project.

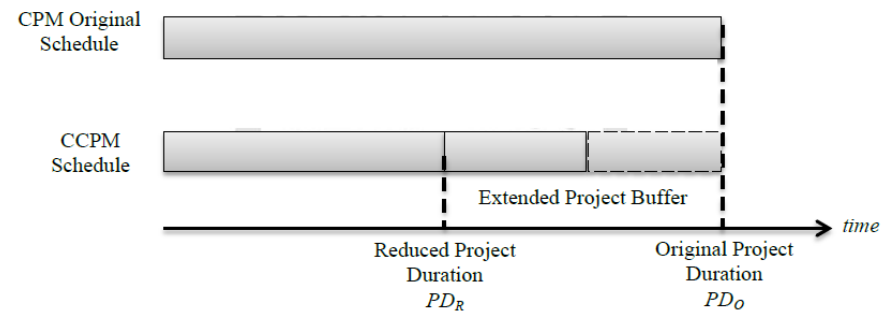

Figure 1. Project Schedule with Extended Project Buffer

Linear Scheduling Method (LSM) is developed to prevent delays or bottlenecks for construction projects on which similar construction activities are execute. LSM allows better representation of scheduling information in terms of space constraints and productivity rates, showing several advantages such as keeping work continuity and maintaining a constant and skilled workforce, which helps to maximize the use of learning curve effect of the crew [2].

Besides the scheduling approaches, some construction management strategies have been developed to manage a project during all the stages of the execution phase. The Earned Value Management (EVM) is a methodology used to measure and communicate the real progress of a project and to integrate the scope, time and cost management. It takes the work accomplished, the time taken and the costs incurred to complete the tasks into account [3]. Moreover, Lean Construction is a methodology designed to produce predictable work flow and rapid learning in scheduling, design, construction and commissioning of projects. The reviewed literature shows that some studies have addressed the integration of criticality concepts of CCPM on LSM in order to define a final schedule in the planning phase. Furthermore, there is a need to develop a cost and time management system which controls that a linear project is developed within budget and schedule previously established during all the stages of the execution phase.

\section{The Proposed Framework for planning and controlling a linear construction project}

Figure 2 shows the framework proposed in this study. The framework seeks to define the steps to follow for a general contractor to manage a construction project since the project is awarded until its total completion based on the lean construction philosophy.

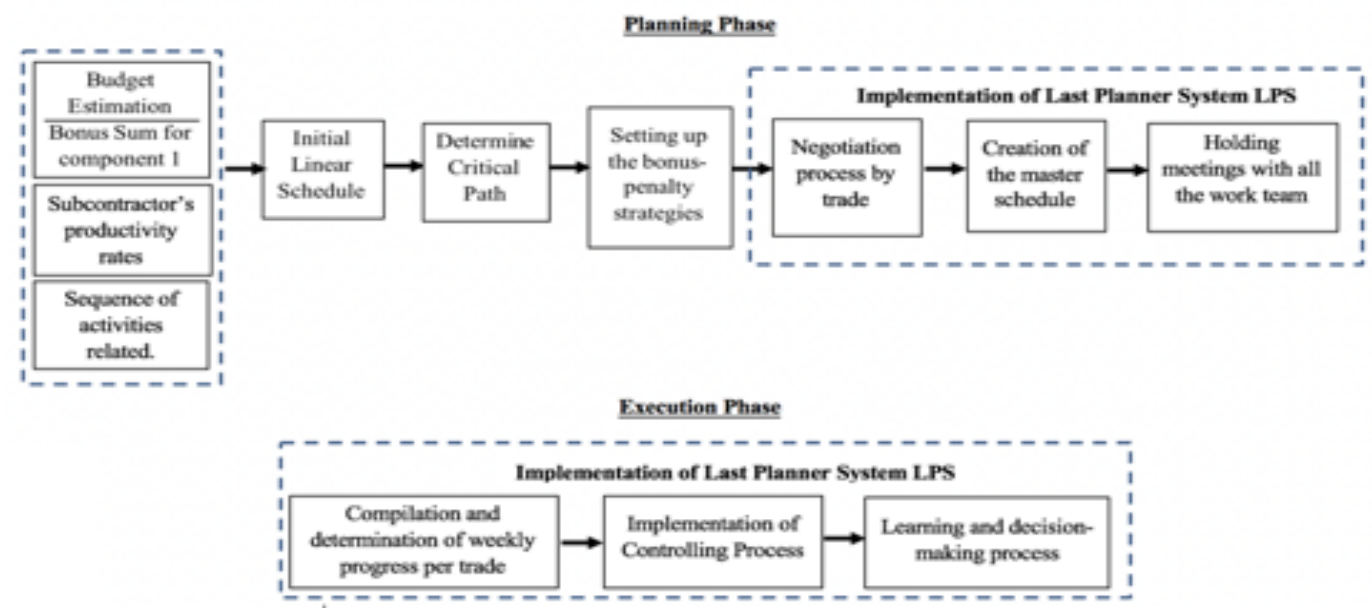

Figure 2. Proposed framework

In terms of time, the linear schedule needs to be created and modified by the duration reduction of some activities in order to create a project buffer allocated at the end of the schedule. Moreover, the feeding buffer for non-controlling segments of the project needs to be determined to avoid new activities become part of the critical path while the project is underway. In terms of cost, a bonus-penalty system and a cost buffer are set along with the traditional cost components in order to develop a cost management system for the execution phase. The time-cost tradeoff strategy together with the Earned Value Management (EVM) are in charge of monitoring and controlling the cost buffer, the 
bonus earned and the real time reduced by the crashed activities in order to determine the status of the project on a weekly basis in terms of time and cost. Each stage and process are explained in the following sections.

\subsection{Planning Phase}

\subsubsection{Initial Linear Schedule and Critical Path}

This study incorporates the concept of the control points developed by Harris and Ioannou in 1998 [4] for finding the critical path. The control points determine the amount of project units that need to be completed in a preceding activity in order to continue with the succeeding activity. Figure 3 shows the two types of control points that can define the critical path of a project in terms of the way the preceding and succeeding activities interact between each other. A control point for start (CPs) occurs when the production rate of the successive activity is higher than the one from the predecessor. This control point is defined between the first project units of the predecessor activity and the successor. On the other hand, a control point for finish (CPf) arises when the production rate of the successive activity is lower than the production rate of the predecessor. This causes that a control point for finish is defined when all the project units of the predecessor are completed. Once all the control points between all the activities are defined, the critical segments are determined in terms of the sequence of the activities established and the rates of their productivities. In Figure 3, the thick lines delimit the critical path comprised of the controlling segments of the project that determine the project duration.

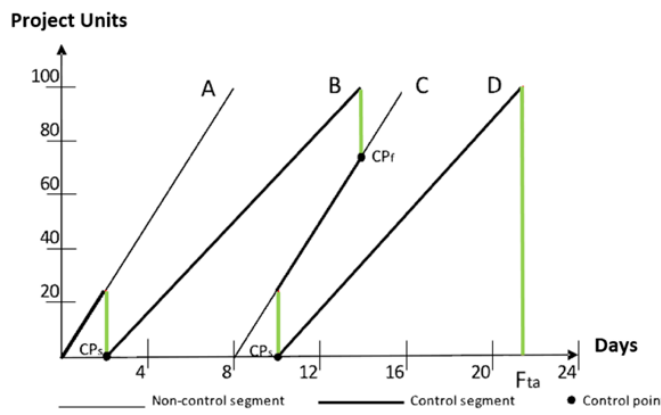

Figure 3. Critical Path Definition by determining the control points

\subsubsection{Setting a bonus-penalty system}

Once the critical path of the initial linear schedule is defined, it proceeds to set the tradeoff strategies for each subcontractor. These strategies are based on offering an attractive incentive (bonus) in return of reducing the duration of its activities. Reducing activity durations in LSM can be done by increasing or making equal the productivities of selected activities and therefore, increasing the slope of the line for the activity [5]. In LSM this can be accomplished by demanding a higher productivity from one crew or by increasing the number of crews [6]. In order to establish the strategies, it is first necessary to define the cost allocation involved for the project. Figure 4 describes the cost breakdown structure taken as the basis for the cost allocation in this framework, which is basically the traditional decomposition of costs, plus the addition of the bonus item.

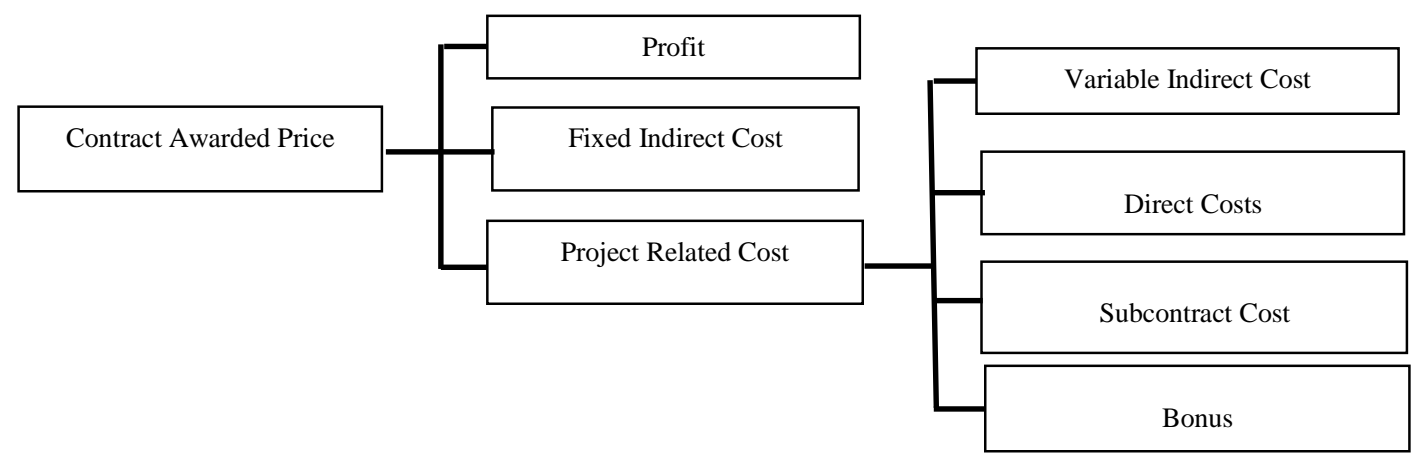

Figure 4. Cost Breakdown Structure 


\section{Bonus}

The main idea of this component is to define a bonus rate, a fixed amount of money per time unit that any subcontractor can get if and only if the activities durations are reduced, regardless of their critical or non-critical status. Therefore, in order to define this bonus rate, the maximum amount that can be actually paid as bonus and the number of days required to be reduced. In Figure 5, available sum (SCAi) represents the original cost component established in the contractual price signed between the project owner and the general contractor, for a contracted activity "i", while referential bidding price (SCRi) represents the cost component established in bid between the general contractor and the subcontract for the same activity " $\mathrm{i}$ ". The bonus component per activity is calculated as follows[7]:

$$
B A_{i}=S C A_{i}-S C R_{i}
$$

$B A_{i}$ : Bonus amount available for trade i. $S C A_{i}$ : Available amount for trade i. $S C R_{i}$ : Referential bidding price agreed between general contractor and trade.

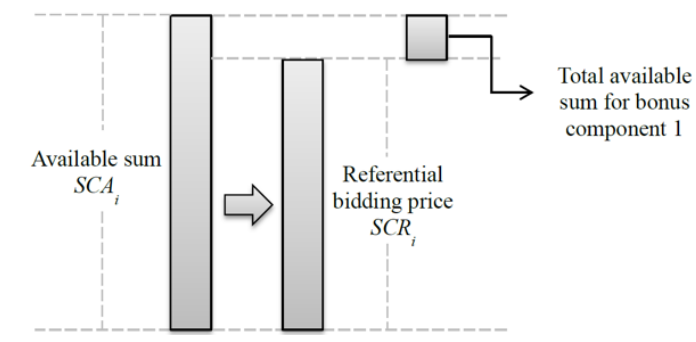

Figure 5 Bonus-Based Strategy for Accelerating Activities

After having determined the maximum amount that can be effectively paid as bonus for each subcontractor, it is time to estimate a rate per time unit. The trade off intends to define of how much the general contractor can actually ask subcontractor to reduce, which is strongly related to how much can be paid as bonus; hence this analysis must be done per each trade involved in the project. The bonus rate per time unit that the trade i can get by reducing one time unit can be determined as follows:

$$
B i=\frac{B A_{i}}{r_{i} x D_{i}}
$$

$B i$ : Bonus rate per time. $r_{i}$ : Percentage of reduction of activity duration i. $D i$ : Duration of activity i corresponding to trade i.

\subsubsection{Negotiation process by trade}

The general contractor convenes meetings with stakeholders separately once the time-cost trade off strategy per trade has been set in order to reduce the duration of its activities. The process involves two steps as described below.

Ask the feasibility of improving productivity rates: The meeting should start by letting each key subcontractor know the need to reduce the duration of its activity (improve its global productivity rate) in order to reduce the project duration, and eventually create the project buffer. This is done by introducing to the representative of the trade (the subcontractor) the existence of a bonus to earn if the goals are achieved. At the same time, the general contractor should listen and take into account any concerns the subcontractor may have.

Negotiate the activity duration reduction: Once both parties have explained their reasons, the negotiation process takes place. In this process, the general contractor must propose the time cost trade off strategy previously set up to the subcontractor. This strategy needs to be discussed together in order to agree the bonus rate per time that will govern in the development of the activity. In other words, a final productivity rate is set between the general contractor and the subcontractor in question, called Determined Productivity $\left(P_{D}\right)$ in this framework. This is the productivity that the general contractor will demand from the subcontractor during all its participation on the project.

\subsubsection{Creation of the Master Schedule}

Once all the time-cost trade off strategies have been agreed between stakeholders and the total reducible time per trade is known, it proceeds to elaborate the final linear master schedule. This process initially includes the determination of the project buffer, then it is necessary to identify the critical and non-critical segments comprising the scheduling. Finally, the feeding buffers of non-critical segments are determined in relation to the type of relationships being formed with the rest of activities. 


\section{Project Buffer}

In linear schedule, selecting the right activities to accelerate in a repetitive project is a very important step toward successful project acceleration [2]. Additionally, a negotiation process between each subcontractor selected to accelerate and the general contractor needs to take place in order to agree the bonus rate per time that will govern in the development of the activity. Once all the time-cost trade off strategies have been agreed between stakeholders and the total reducible time per trade is known, it proceeds to elaborate the final linear master schedule. This process initially includes the determination of the project buffer. Figure 6 shows an example for a linear schedule with 4 different activities (A, B, C and, D). The relationship between activities indicates that activity $\mathrm{A}$ and $\mathrm{C}$ have the same productivity rate. Also shows that activity $\mathrm{B}$ has a lower productivity than its predecessor activity A. Activity $\mathrm{C}$ has a higher productivity than its predecessor B and successor D. Moreover, the end of the project corresponds to the original project duration (PDo). From Figure 3, it can be seen that activity B and D have the lowest productivity rates and therefore, they have a high influence over the project duration. Figure 6 shows the same set of activities from figure 3 but with a notable increment of productivity rate $\mathrm{B}$. This causes that the relationship among activities change a little and therefore, the end of the project corresponds to new crashed duration of the project (NCD). The time difference between PDo and NCD creates the size of the initial project buffer (IPB).

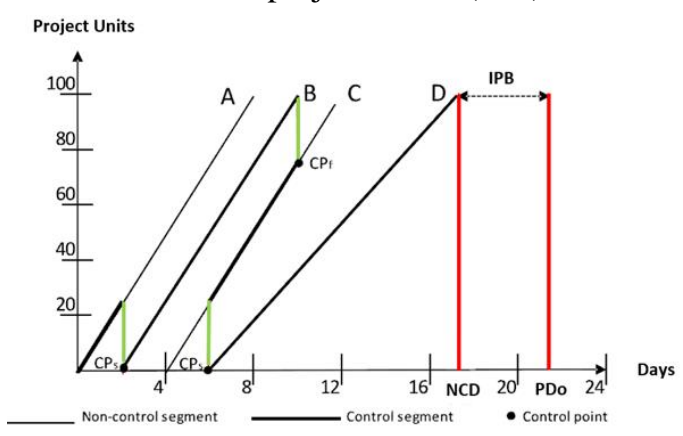

Figure 6 Linear Schedule after applying time-cost tradeoff over activity B

\section{Cost Buffer and Cost Buffer Allocation}

This study proposes the inclusion of a cost buffer in order to manage the uncertainty of the cost estimate when developing a budget in the planning stage. The cost buffer is usually used for dealing with unexpected events during the construction process in critical activities. For this research, the total cost buffer of the project was considered as the amount of variable indirect cost designated for the time units that represent the initial project buffer (IPB). Therefore, the total cost buffer available for a project $\left(\mathrm{CB}_{\mathrm{T}}\right)$ includes the variable indirect cost rate $\left(\mathrm{VIC}_{\mathrm{R}}\right)$ per time unit with the total duration of the initial project buffer (IPB). $\mathrm{CB}_{\mathrm{T}}=\mathrm{VIC}_{\mathrm{R}} * \mathrm{IPB}$.

It was defined according to the assumption that the variable indirect cost (VIC) is associated to the subcontractor cost of the critical segments $\left(\mathrm{SDC}_{\mathrm{C}-\mathrm{Chain}}\right)$. This association is due to both cost components have a direct relationship with the project duration because the critical chain segments of the linear schedule control the project duration and the total variable indirect cost spent in a project directly depends on the project duration. Furthermore, this research includes a new approach for monitoring the interaction between the bonuses earned with the cost buffer consumed. The cost buffer is only allocated to the controlling segments of the project. The allocation process starts with the partition of the project into sections bounded by the controlling segments. For each section, the percentage of the budget that represents is estimated by including all the subcontractor direct cost for the different subcontractors (SDC) and the variable indirect cost scheduled in the master. Later, the allocation for each section takes place by multiplying the weighted values with the total cost buffer amount. Finally, it is necessary to determine the total cost buffer amount for each section in a cumulative way. Different sections in the project is defined to include all the cost assigned to the scheduled works of the different trades within the development of each of the controlling segments. For each section, the total cost is calculated to determine how much weight it has in the total cost of the project (weighted value) to finally allocate the cost buffer in a proportional way.

\section{Determination of feeding buffers in the critical path}

In the case of linear schedule, the relationship between one activity and another can be seen in terms of repetitive number of units or "batches", on which the start of a batch from a succeeding activity is conditioned by the completion of a preceding batch (Shim and Yoo, 2013).[9] Figure 7(a) shows in green the controlling batches, and in light blue 
the non-controlling batches for a linear schedule consisting of four activities. Figure 7(b) shows the relationship between activity $\mathrm{A}$ and $\mathrm{B}$ in terms of the interaction of their controlling and non-controlling batches. From the relationship can be seen that the first batch of activity B (1B) only can start when the first batch of activity A is completed. Also, it can be observed that the productivity rate of the predecessor activity (A) is higher than the successor activity (B), which causes that the non-controlling batches of A (2A, 3A, and 4A) still have float time from their scheduled finish time until the scheduled start of the controlling batches from activity B (2B, 3B, and 4B). These float times calculated per batch become the feeding buffers of the non-controlling segments of activities that at least have a segment within the critical path. For instance, the feeding buffer of the second batch of activity A respect to the second batch of activity $\mathrm{B}$ is calculated using the following expression:

Feeding buffer of batch $2 \mathrm{~A}$ respect to batch $\mathrm{AB}$ (FB1)

Scheduled completion time of $2 \mathrm{~A}=\mathrm{TF}-2 \mathrm{~A}$

Scheduled start time of $2 \mathrm{~B}=\mathrm{T}$ S-2A FB1 $=$ TS-2A - TF-2A

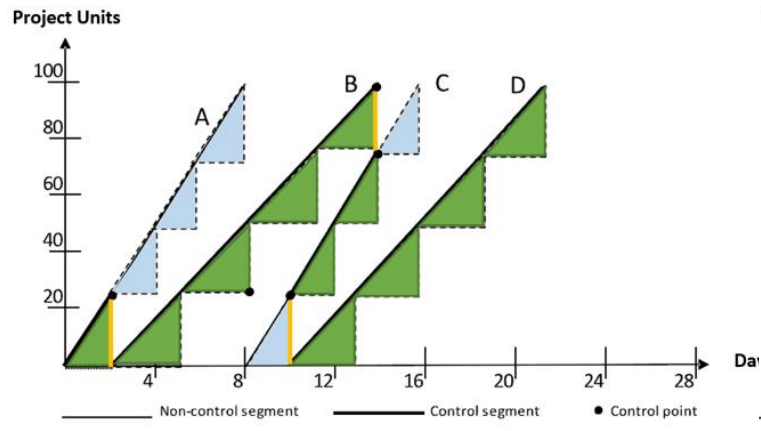

(a)

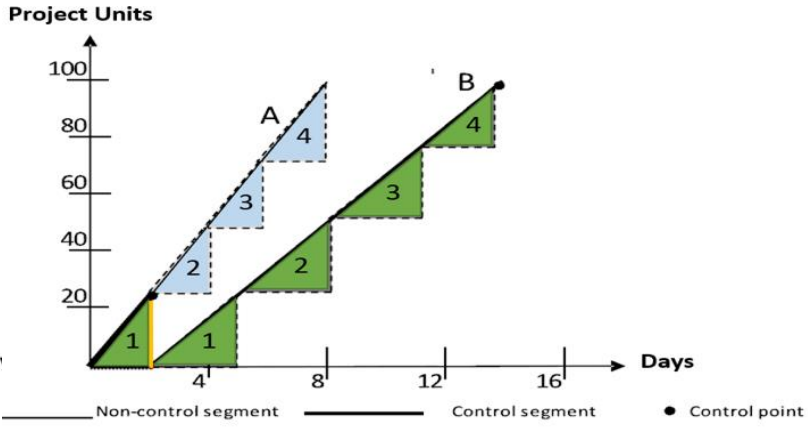

(b)

Figure 7 Relationship between Control and Non-Control Batches of two activities

It is important to mention that the feeding buffers in the non-critical chain also need to be determined according to the type of relationship that each of those activities interacts with either critical or non-critical activities. Finding buffers of the non-controlling segments seeks to monitor and to prevent this segment to become critical in the future.

\subsection{Monitoring and controlling the status of the project}

The second section of Figure 2 depicts the set of steps proposed in this study is to monitor and control the development of the project. In summary, the steps initially include that subcontractors already in operations are required to report their weekly performance at the end of each work week. Then, with the information retrieved the general contractor has to develop the controlling process that includes the determination of the progress of each trade, the project buffer consumption, the cost buffer consumption and the evaluation of the indices obtained. Moreover, finishing the controlling process the general contractor must convene a meeting with stakeholders in order to define the actions required to keep developing properly the works for the next week. Each part of the process is explained in more detail below.

\subsubsection{Determination of progress of each trade, \% Complete}

Based on the information reported by each subcontractor currently working in the project, it is estimated the percentage of progress each trade has obtained. This study considers the participation of two types of subcontractors in terms of the way their performance can be measured in a more accurate way.

For the trades (TD) in charge of works in which all their progress can be well represented in a single unit of measure, the \% Complete is calculated from the relation between the cumulative units of measure of the project already completed $\left(\mathrm{UC}_{(\mathrm{TD})}\right)$ and the total units of measure of the project $\left(\mathrm{UT}_{(\mathrm{TD})}\right)$.

$$
\% \text { Complete }=\frac{U_{C(T D)}}{U_{T(T D)}} * 100
$$




\subsubsection{Determination of Project Buffer Consumption}

The applicability of the Project Buffer Consumption methodology is just focused on the critical segments that control the total project duration. In other words, through the use of the control points between subcontractors previously determined at the planning stage, it is possible to apply the methodology on a linear scheduling. The process is based on determining whether the project is running behind or ahead schedule. If the project has experienced some delays during the week, it is necessary to determine how many days the project is behind the master schedule. First of all, it is estimated the real date the units associated to the critical segment will be completed for the trade. This is obtained by subtracting from the total quantity of its critical units ( $\left.\mathbf{U}_{\mathbf{T C S}}\right)$, the units of work completed so far ( $\mathrm{U}_{\mathrm{CCS}}$ ), as well as by assuming the critical trade in question will work at a constant contractual productivity (determined productivity, $\mathrm{P}_{\mathrm{D}}$ ) from the date of analysis until the remaining units belonging to the critical segment are done. Finally, the estimated date of completion is compared with the scheduled date the critical segment should have been completed $\left(\mathrm{T}_{\mathrm{ED}}\right)$. The project buffer consumption can be determined as follows.

$$
P B_{C M}=\left[N+\frac{U_{T C S}-U_{C C S}}{P_{D}}\right]-T_{E D}
$$

Then, the percentage of project buffer consumption in relation to the initial project buffer time (IPB) is determined, as follows.

$$
\% P B_{C M}=\frac{P B_{C M}}{I P B}
$$

\subsubsection{Determination of Total Cost and Cost Buffer Consumption}

\section{Total Cost, $A C$}

The first step is the determination of the actual cost that the project is having until the date of analysis. Its determination includes the calculation of several cost components for each of the subcontractors currently working on the project. Below is explained in detail how the process is carried out. For each trade has to be calculated the subcontractor direct cost (AC $\mathbf{A D C})$ that represents the direct cost to be paid to the subcontractor by work done. Likewise, the bonus earned (BE) for the subcontractor is calculated if only if the percentage of progress $\%$ complete reaches a 100 value. Obviously, the amount of bonus earned depends on the difference between the real date of completion ( $\mathbf{T}_{\mathbf{R D T O T A L}}$ ) with the planned date of completion of the works ( $\mathbf{T}_{\mathbf{P D T O T A L}}$ ) by the trade in question. Table 2 shows an example of how a trade is monitored on a weekly basis within this framework from the beginning to the end of its participation in the project. Furthermore, the Actual Variable Indirect Cost (ACvic) needs to be calculated on a weekly basis. It comes from the current project duration $(\mathrm{N})$ and the variable indirect cost rate $\left(\mathbf{V I C}_{\mathbf{R}}\right)$ defined by the general contractor prior the beginning of the execution process. Table 3 shows the table format proposed for the determination of this cost component. Then, the total Actual cost $\left(\mathbf{A C}_{\mathbf{T}}\right)$ is estimated through the summation of the actual subcontractor direct cost of all the trades ( $\mathbf{A} \mathbf{C}_{\text {SDC }}$ cum), the actual variable indirect cost (AC $\mathbf{C}_{\text {VIC }}$ ), and the bonus earned (BE) for the subcontractors that already complete their works.

\section{Cost Buffer Consumed, $\mathrm{CB}_{C M}$}

The second step corresponds to the determination of how much cost buffer has been consumed so far. Below is explained in detail how the process is carried out. First of all, it is necessary to calculate the earned value of the project he calculation of the consumption of the cost buffer includes the calculation of the cost variance of the project (CV) by comparing the budgeted cost performed with the actual cost incurred in the project without including the bonus earned. The following expression shows the way the $\mathbf{C V}$ is calculated weekly.

$$
C V=E V-\left[A C_{\text {TOTAL-WEEK } n}-B E_{T}\right]
$$

Then, the determination of the cost buffer consumed to week of analysis comes from the assessment of the weekly cost variance value. Table 4 shows the assessment of the weekly cost variance value obtained.

\begin{tabular}{|c|c|l|}
\hline If $\mathrm{CV}>0$ & $C B_{C M}=0$ & There is no consumption of cost buffer. \\
\hline If $\mathrm{CV}<0$ & $C B_{C M}=|C V|$ & $\begin{array}{l}\text { Cost buffer is being consumed. } \\
\text { Needs to be evaluated if the consumption is within allowable } \\
\text { limits to determine if the cost is over budget or not. }\end{array}$ \\
\hline
\end{tabular}




\section{Conclusions}

The framework proposes a methodology for the general contractor to plan and control a linear construction project. It includes the steps necessary to manage a project from the planning stage to execution. The development of the project schedule is carried out based on the integration of the concepts of the Critical Chain Project Management on the Linea Scheduling Method in order to provide cohesion and continuity in projects developed under the linear approach. The implementation of a bonus-penalty system seeks to reduce the duration of certain project activities by increasing the productivity rates of the subcontractors in charge of them, while they are given the chance to win a bonus. The establishment of a project buffer that is allocated at the end of the project gives the general contractor a mechanism to control the critical chain in terms of the contractual date of completion.

Methodologies like the Buffer Management and the Project Buffer Index are also included in the implementation of the monitoring process of the project buffer. Their contribution falls on the establishment of the criteria that define the status of the project in terms of buffer consumption and the percentage of progress of the critical chain. The Earned Value Management has been addressed within the framework for developing the cost management process. This process involves the evaluation and control of the cost buffer consumption by measuring project progress in monetary terms. In other words, the cost management is based on constantly monitoring the "Cost-Buffer Consumed - Bonus Earned" interaction because, as long as the bonus available for each trade is totally or partially earned by the subcontractors who have done a good performance along the project, the Cost Buffer consumed will be within the allowable limits given by the budget. The importance of creating these control points is to control that non-critical batches never become critical along the project and subsequently, extend the project duration. However, during the execution phase the feeding buffers of some non-critical segments need to be updated as long as the critical segments they have relationship with, have delays and consume project buffer. Finally, it is worth noting the Lean Construction concept is the philosophy that governs the framework of this research. This concept is included in all phases of the project. During the planning phase, it is applied to empower the integration of all project stakeholders in order to develop an efficient and achievable final master schedule. On the other hand, during the execution phase, Lean Construction philosophy seeks to improve the coordination among all project participants as well as their performance by conducting weekly meetings.

\section{References}

[1]E. Goldratt, Critical chain. Great Barrington, MA: 1997 North River Press. ISBN-10: 0884271536. ISBN-13: 978-0884271536.

[2]L. Bakry, O. Moselhi, T. Zayed, Optimized acceleration of repetitive construction projects. Automation in Construction, 2014, 39, 145-151. doi:10.1016/j.autcon.2013.07.003.

[4] R.B. Harris, P.G. Ioannou, Scheduling Project with Repeating Activities, J. Constr. Eng. Manage., 1998, 124(4): 269-278

[3]M, Vanhoucke, Project Management with Dynamic Scheduling: Baseline Scheduling, Risk Analysis and Project Control. Springer. 2013, $2^{\text {nd }}$ edition. Pages 202-205.

[5] A. Marín, 2015. The Framework of Applying the Critical Chain to Linear Schedule for Construction Project Management. National Cheng Kung University, 2015 Mater Thesis, Taiwan.

[6] A. Hassanein, O. Moselhi, Planning and Scheduling Highway Construction J. Constr. Eng. Manage., 2004, 130(5): 638-646

[7] G. Andrade, 2012. Framework for Adapting Critical Chain Methodology to Construction Project Management. National Cheng Kung University 2013, Mater Thesis, Taiwan. 